Original Article

\title{
Aetiological Evaluation of Patients with Meningitis in a Tertiary Care Hospital of Bangladesh
}

\author{
${ }^{*}$ Islam $S^{1}$, Hasan $M N^{2}$, Hoque $M A^{3}$, Shahi $M S^{4}$, Rahman $M A^{5}$, Hasan $M^{6}$
}

\begin{abstract}
Meningitis is a global health problem as mortality is high and a large proportion of survivors suffered from significant morbidity. The physicians are facing this challenge of emergency identification of this clinical syndrome, establishing its etiology and its prompt treatment not only to ensure survival but also to prevent long term sequelae in these patients. Poor outcomes caused by bacterial meningitis due to delays in diagnosis and treatment. In Bangladesh, the epidemiological study regarding meningitis in adults is rare. Rapid o easily available as well as specific test or means are also not in our hand. The aim of this study was to evaluate the aetiology of patient with meningitis in tertiary care hospital of Bangladesh. The main objective was to evaluate the aetiology of meningitis of patients admitted in medical wards of a tertiary care hospital. The study was a descriptive type of observational study. The study was carried out in the Department of Medicine, Dhaka Medical College Hospital, a tertiary level hospital of Bangladesh from first July 2013 to thirty first December 2013. A total 50 patients were enrolled with Meningitis, diagnosed on clinical, biochemical, other investigational background, fulfilling the inclusion and exclusion criteria.Patients having feature of meningitis was enrolled in the study after getting informed written consent from patient or attendant. Detail demographic data were collected
\end{abstract}

1. ${ }^{*}$ Dr. Sadia Islam, Assistant professor ,department of medicine. Delta medical college and hospital,Dhaka. Email: sadia_8899@yahoo.com

2. Dr. Md. Nazmul Hasan, Assistant professor, Department of Internal Medicine. Bangabandhu Sheikh Mujib Medical University, Dhaka-1000

3. Dr .Md. Azharul Hoque, Professor Department of neurology. National institute of neuroscience

Dhaka ,Bangladesh

4. Dr. Mohammad Selim Shahi, Associate professor. Department of neurology.National institute of neuroscience, Dhaka, Bangladesh

5. Dr. Md. Atikur Rahman, Resident phase-B, Department of Internal Medicine, Bangabandhu Sheikh Mujib Medical University,Dhaka-1000

6. Dr. Mehedi Hasan, Medical officer,department of anaesthesia,analgesia, and ICU, Bangabandhu Sheikh Mujib Medical University, Dhaka-1000

${ }^{*}$ For Correspondence from the informant was recorded in structured case report form. Clinical examination and relevant investigation with CSF study were done. Routine follow up of the patient was done. Data analysis was conducted with SPSS software. Among the 50 patient the mostly affected groups were below 40 years of age comprising $66 \%$. The mean $( \pm S D)$ age was $33.04 \pm 18$ years. There was an overall male preponderance with a male to female ratio 1.63:1 ( $N=31$ vs $N=19)$. The highest number of patients were presented with fever (100\%), headache (98\%) and altered mental status (88\%). Among all 50 cases of meningitis $27(54 \%)$ were diagnosed as pyogenic, 9 (18\%) were tuberculous meningitis and 14(28\%) were viral . Out of 27 pyogenic meningitis cases 19 (70.37\%) developed convulsion, out of 9 tuberculous meningitis 3 (33.33\%) developed convulsion and 11 (78.57\%) out of 14 viral cases had convulsion, that is convulsion was more common in viral cases. Sixteen male patients (51.61\%) and 11 female patients (57.89\%) had pyogenic meningitis, 5 male patients (16.12\%) and 4 female patients (21.05\%) had tuberculous meningitis. On the other hand, 10 (32.25\%) male and 4 (21.05\%) female had viral meningitis. P value was 0.369, So the male and female difference was not statistically significant. Diagnosis was made on the basis of clinical findings and CSF study. Pyogenic were more common than viral cases and tubercular meningitis. Meningitis is foremost causes of morbidity and mortality. It is recommended that provision of proper health care support, Proper and rapid detection and others investigation facilities reduce the disability. In the interim, this study provides data that can inform public health strategies directed at assessing and reducing meningitis severity and meningitis events.

Key words: Meningitis, Aetiology, CSF study

\section{INTRODUCTION}

Meningitis is an acute inflammation of the protective membranes covering the brain and spinal cord, known collectively as the meninges. ${ }^{1}$ The inflammation may be caused by infection with viruses, bacteria, or other microorganisms, and less commonly by certain drugs. ${ }^{2}$ Meningitis can be life-threatening because of the inflammation's proximity to the brain and spinal cord; therefore, the condition is classified as a medical emergency.

Although meningitis is a notifiable disease in many countries, the exact incidence rate is unknown. ${ }^{3}$ As of 2010 it is estimated that it resulted in 420,000 deaths. Bacterial meningitis occurs in about 3 people per 100,000 annually in 
Western countries. Population-wide studies have shown that viral meningitis is more common, at 10.9 per 100,000 , and occurs more often in the summer. In Brazil, the rate at 45.8 per 100,000 annually. ${ }^{4}$ Sub-Saharan Africa has been plagued by large epidemics of meningococcal meningitis for over a century, leading to it being labeled the "meningitis belt". ${ }^{5} \mathrm{In}$ Bangladesh major etiological factors revealed that $18 \%$ are meningococcal, $3 \%$ pneumococcal, and 3\%Hib infection. Twenty percent of patients with Hib meningitis are older than 15 years. Case-fatality ratios $10 \%$ for N. meningitid is, $22 \%$ for S. pneumoniae, and $24 \%$ for Hib. Bacterial meningitis from vaccine-preventable pathogens causes significant morbidity and mortality in Bangladesh in adults and children. ${ }^{6}$

Epidemics typically occur in the dry season (December to June), and an epidemic wave can last two to three years, dying out during the intervening rainy seasons. These cases are predominantly caused by meningococci. ${ }^{4}$ The largest epidemic ever recorded in history swept across the entire region in 1996 to 1997 , causing over 250,000 cases and 25,000 deaths. Several factors have been associated with the development of epidemics in the meningitis belt. They include: medical conditions (immunological susceptibility of the population), demographic conditions (travel and large population displacements), socioeconomic conditions (overcrowding and poor living conditions), climatic conditions (drought and dust storms), and concurrent infections (acute respiratory infections). ${ }^{7,8}$

The infection may trigger sepsis, a systemic inflammatory response syndrome of falling blood pressure, fast heart rate, high or abnormally low temperature, and rapid breathing. Very low blood pressure may occur at an early stage, especially but not exclusively in meningococcal meningitis; this may lead to insufficient blood supply to other organs. Disseminated intravascular coagulation, the excessive activation of blood clotting, may obstruct blood flow to organs and paradoxically increase the bleeding risk. Gangrene of limbs can occur in meningococcal disease. ${ }^{1}$

The brain tissue may swell, pressure inside the skull may increase and the swollen brain may herniated through the skull base. This may be noticed by a decreasing level of consciousness, loss of the pupillary light reflex, and abnormal posturing. The inflammation of the brain tissue may also obstruct the normal flow of CSF around the brain (hydrocephalus). ${ }^{9}$ Seizures may occur for various reasons; in children, seizures are common in the early stages of meningitis (in 30\% of cases) and do not necessarily indicate an underlying cause. Seizures may result from increased pressure and from areas of inflammation in the brain tissue. Focal seizures, persistent seizures, late-onset seizures indicate a poorer long-term outcome. ${ }^{1}$

Meningitis can lead to serious long-term consequences such as deafness, epilepsy, hydrocephalus and cognitive deficits, especially if not treated quickly. Some forms of meningitis (such as those associated with meningococci, Haemophilus influenzae type $B$, pneumococci or mumps virus infections) may be prevented by immunization., ${ }^{1,9}$

Untreated, delay treated, bacterial meningitis is almost always fatal. Viral meningitis, in contrast, tends to resolve spontaneously and is rarely fatal. With treatment, mortality from bacterial meningitis depends on the age of the person and the underlying cause. Of newborns, $20 \%-30 \%$ may die from an episode of bacterial meningitis. This risk is much lower in older children, whose mortality is about $2 \%$, but rises again to about $19 \%-37 \%$ in adults. Risk of death is predicted by various factors apart from age, such as the pathogen and the time it takes for the pathogen to be cleared from the cerebrospinal fluid, ${ }^{1}$ the severity of the generalized illness, a decreased level of consciousness or an abnormally low count of white blood cells in the CSF. Meningitis caused byH. influenzae and meningococci has a better prognosis than cases caused by group B streptococci, coliforms and $S$. pneumonia. In adults, too, meningococcal meningitis has a lower mortality $(3 \%-7 \%)$ than pneumococcal disease. ${ }^{9}$

Management of bacterial meningitis involves several crucial questions regarding the optimal timing of therapy. First, given that the diagnosis of bacterial meningitis can be definitively made only by CSF analysis, does early antibiotic administration before LP affect culture results and thereby compromise targeted therapy? Second, does the performance of diagnostic testing such as computed tomography (CT) and LP significantly delay antibiotic therapy? Lastly, does delayed therapy produce worse clinical outcomes. In a retrospective pediatric study, results confirmed previous suspicions that early antibiotics do, in fact, reduce culture yields: Parenteral antibiotics can complete sterilization of meningococcus in the CSF within 2 hours of therapy and pneumococcus within 4 hours. ${ }^{10}$ However, if the patient's clinical presentation mandates a head CT prior to LP, an average of 6 hours lapses between presentation and parenteral antibiotics.

One of the greatest fears passed down in emergency medicine dogma is the risk of brain herniation upon sudden release of increased intracranial pressure from LP. The true incidence of this devastating complication is unknown and is based mostly on retrospective observational data. ${ }^{11}$ 
Regardless, neuro-imaging should be obtained prior to LP in patients with abnormal neurological exams. The Infectious Disease Society of America (IDSA) recommends obtaining a head CT prior to LP for patients who meet any of these criteria: immunocompromised state, history of CNS disease (mass lesion, stroke, or focal infection), new-onset seizure within 1 week of presentation, papilledema, abnormal level of consciousness, or focal neurologic deficit. ${ }^{11}$

\section{MATERIALS AND METHODS}

The study was a descriptive type of observational study. The study was carried out in the Department of Medicine, Dhaka Medical College Hospital, a tertiary level hospital of Bangladesh from first July 2013 to thirty first December 2013. A total 50 patients were enrolled with Meningitis, diagnosed on clinical, biochemical, other investigational background, fulfilling the inclusion and exclusion criteria. The inclusion criterias were hospital admitted patients in medical wards with fever, headache, and vomiting, meningeal irritation, e.g. neck rigidity, positive Kerning's sign and/or positive Brudzinskis sign, a dult patients of both genders (twelve years and above).

And the exclusion criterias were patients who refuse lumbar puncture or die before doing lumbar puncture,patients of encephalopathy due to metabolic and endocrine causes, patients who have recent head trauma, immune compromised, known malignant lesions, Central nervous system neoplasm Critically ill patient or patient refused to give consent or not able to do CT-scan will be excluded from the study, withdrawal of informed consent. Patients having feature of meningitis was enrolled in the study after getting informed written consent from patient or attendant. Detail demographic data were collected from the informant was recorded in structured case report form. Clinical examination and relevant investigation with CSF study were done. Routine follow up of the patient was done. Data analysis was conducted with SPSS software

\section{RESULTS}

In this series, the maximum numbers of patients were between 13-20 years of age group, 22(44\%). Then 21-30 years of age $8(16 \%)$ (Table 1$)$.Out of 50 cases $31(62 \%)$ were male and $19(38 \%)$ were female and male to female ratio was 1.63:1. Maximum numbers of male and female patients were in age group between 13-20 years. It is about $48.38 \%$ and $36.84 \%$ respectively. Patients came from both urban and rural areas with urban $(66 \%)$ preponderance. (Table-1)
Table 1: Age distribution of patient. $(n=50)$

\begin{tabular}{|l|c|c|c|}
\hline $\begin{array}{l}\text { Age } \\
\text { (years) }\end{array}$ & $\begin{array}{c}\text { Number of } \\
\text { patients }\end{array}$ & Percentage (\%) & Mean \pm SD \\
\hline $13-20$ & 22 & 44 & \multirow{2}{*}{$33.04 \pm 18.08$} \\
\hline $21-30$ & 8 & 16 & \\
\hline $31-40$ & 3 & 6 & \\
\hline $41-50$ & 3 & 6 & \\
\hline $51-60$ & 6 & 12 & \\
\hline$>60$ & 8 & 16 & \\
\hline
\end{tabular}

Among the patients the poor class 22(44\%) comprising the major percentage of the meningitis patients, which is followed by middle class $19(38 \%)$ and remaining are upper class 9(18\%). (Figure 1)

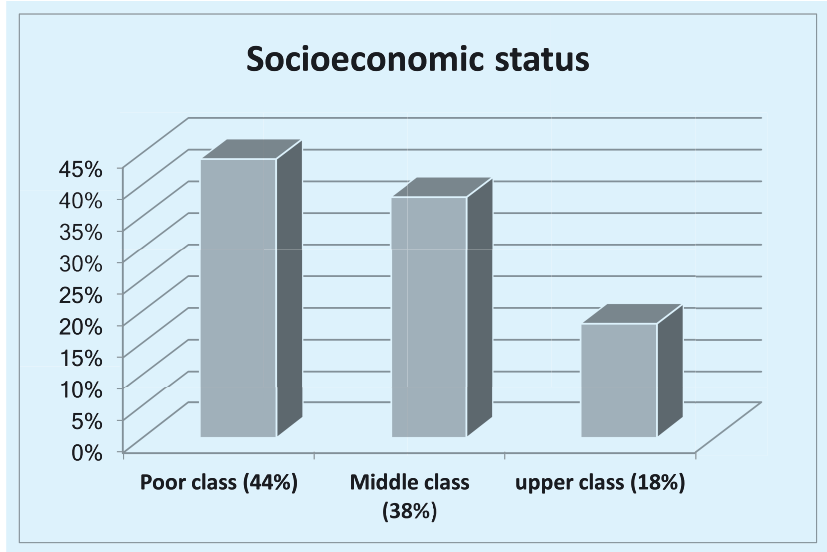

Figure- 1: Socioeconomic status.

In this series, the maximum number of patients $50(100 \%)$ faced their crisis with acute onset fever, and then 49 (98\%) of cases presented with headache, then altered mental status $44(88 \%)$ of cases . About 50\% patients (25) were Lethargic and $32 \%$ patients were comatose. The difference was statistically not significant $(P>0.05)$.In this series, the maximum number of patients $50(100 \%)$ presented with neck rigidity, and then kernig's sign 43 (86\%), abnormal pupil $62 \%$ and extensor plantar response $40 \%$ of patient.(Table-2)

Table- 2: Distribution of patients according to symptoms and signs of meningitis. $(n=50)$

\begin{tabular}{|l|c|c|}
\hline Presentation & Number of patients & Percentage (\%) \\
\hline Fever & 50 & $100 \%$ \\
\hline Altered mental status & 44 & $88 \%$ \\
\hline Headache & 49 & $98 \%$ \\
\hline New onset seizure & 11 & $22 \%$ \\
\hline Vomiting & 31 & $62 \%$ \\
\hline Restlessness & 29 & $58 \%$ \\
\hline Photophobia & 21 & $42 \%$ \\
\hline Neck rigidity & 50 & $100 \%$ \\
\hline Kernig's sign & 43 & $86 \%$ \\
\hline Abnormal pupil & 30 & $60 \%$ \\
\hline Extensor plantar response & 20 & $40 \%$ \\
\hline
\end{tabular}


Among the 50 cases of meningitis $27(54 \%)$ cases were diagnosed as pyogenic meningitis. Out of 31 male patient $16(51.61 \%)$ had pyogenic, $5(16.12 \%)$ tuberculous and 10 (32.25\%) had viral causes. In case of female $11(57.89 \%)$ had pyogenic, $4(21.05 \%)$ tuberculous and $4(21.05 \%)$ had viral meningitis. The difference was statistically not significant $(\mathrm{P}>0.05)$. $($ Table- 3$)$

Table- 3: Distribution of cases according to aetiological factors. $(n=50)$

\begin{tabular}{|l|c|c|c|}
\hline Type of meningitis & \multicolumn{2}{|c|}{ Number of patients } & Total \\
\hline & Male $(\mathrm{n}=31)$ & Female $(\mathrm{n}=19)$ & \\
\hline Pyogenic meningitis & $16(51.61 \%)$ & $11(57.89 \%)$ & $27(58 \%)$ \\
\hline Tuberculous meningitis & $5(16.12 \%)$ & $4(21.05 \%)$ & $9(18 \%)$ \\
\hline Viral meningitis & $10(32.25 \%)$ & $4(21.05 \%)$ & $14(28 \%)$ \\
\hline
\end{tabular}

The convulsions were less common in viral cases $11(22 \%)$ cases compared to pyogenic meningitis which was $38 \%$. The difference was statistically not significant $(\mathrm{P}>0.05) .($ Table- 4$)$

Table-4: Association with seizure/convulsion. ( $n=50)$

\begin{tabular}{|l|c|c|c|}
\hline Type of meningitis & $\begin{array}{c}\text { Convulsion } \\
\text { present }\end{array}$ & $\begin{array}{c}\text { Convulsion } \\
\text { Absent }\end{array}$ & P value \\
\hline Pyogenic & 19 & 8 & 0.242 \\
\hline Tuberculous meningitis & 3 & 6 & \\
\hline Viral meningitis & 11 & 3 & \\
\hline Total & 33 & 17 & \\
\hline
\end{tabular}

\section{DISCUSSION:}

Our study design raises a number of important methodological issues, including patient selection, sample size and the prospective identification of case according to clinical and pathological evaluation, all of which may exert a powerful influence on the results. These issues may be particularly relevant for observational studies that use aetiology, risk, complication as an outcome measure, in which sources of bias may be sufficiently large to either obscure a real difference in rates or create an apparent one. Using our results and previous studies as examples, we shall address these issues in turn.

Most of the published studies on meningitis have focused on individual problems in isolation, such as causes or clinical manifestation e.g. seizures, acute onset fever, neck rigidity or feature of meningeal irritation. These studies have used a range of different designs; furthermore, methods of patient selection, diagnostic criteria, timing, and duration of follow-up vary considerably between studies, and therefore it is hardly surprising that the reported frequencies of specific complications in these studies also varied.
Infection of the central nervous system is a medical emergency. Fifty consecutive cases (age above 12 years) were recruited. The mostly affected groups were below 40 years of age comprising 66\%. 22 cases (44\%) were in 13-20 years age group and also there was a small rise in elderly patients ( 8 cases, 16\% above 60 years of age). Mean age was 33.04 $( \pm 18.08 \mathrm{SD})$ years which correlates with another study done in India where mean age was $32.54 \pm 13.32$ years (range from $15-70$ years) of which $56 \%$ were male and $44 \%$ were female. ${ }^{12}$ In a hospital based prospective study ${ }^{13}$ done in Bangladesh (2003-2005) for etiology of bacterial meningitis $66 \%$ adult patients were in 15-36 years age group. In AMES surveillance14 study in Bangladesh from 2007 to 2009 mean age of Japanese encephalitis patients was 31 years ranging from 1 month to 85 years and mean age of total AMES patients was 18 years. There was an overall male preponderance with a male to female ratio $1.63: 1(\mathrm{~N}=31 \mathrm{vs}$ $\mathrm{N}=19$ ). This finding has similarity with a prospective clinical study published in Arch Neurol Journal in 1993. ${ }^{15}$

In this series ${ }^{13}$ maximum patients were presented with fever $(100 \%)$, headache $(98 \%)$ and altered mental status $(88 \%)$. Out of 50 cases $11(22 \%)$ patients developed new onset seizure and $30(60 \%)$ developed signs of meningeal irritation. 25 patients (50\%) were lethargic and $16(32 \%)$ cases were comatose. Female were more comatose $(36.84 \%)$ compared to male (29\%). It correlates with hospital based prospective study of Bangladesh. In a nation wide study done in Netherlands to determine clinical features and prognostic factors in adult with community acquired acute meningitis from October 1998 to April 2002, 95\% patients had at least two of four symptoms of headache, fever, neck stiffness and altered mental status. ${ }^{16}$

Out of 27 pyogenic meningitis cases 19 (70.37\%) developed convulsion, out of 9 tuberculous meningitis 3 (33.33\%) developed convulsion and 11 (78.57\%) out of 14 viral cases had convulsion, that is convulsion was more common in viral casesStudy showed clinical presentation of mental status among the meningitis patient. Out of 14 viral meningitis cases 6(42.85\%) were comatose state, $10(37.03 \%)$ pyogenic cases were comatose and there is no coma in tuberculous meningitis. That is unconsciousness was more common in viral group. This finding correlates with another study. ${ }^{18}$

Among all 50 cases of meningitis 27(54) were diagnosed as pyogenic, $9(18 \%)$ were tuberculous meningitis and $14(28 \%)$ were found viral aetiology. 16 male patients (51.61\%) and 11 female patients $(57.89 \%)$ had pyogenic meningitis, 5 male patients $(16.12 \%)$ and 4 female patients 
(21.05\%) had tuberculous meningitis. On the other hand, $10(32.25 \%)$ male and $4(21.05 \%)$ female had viral meningitis. P value was 0.369 , So the male and female difference was not statistically significant. Diagnosis was made on the basis of clinical findings and CSF study (Cytology, biochemistry, Latex agglutination test). Pyogenic were more common than viral cases.

Among the 50 cases of meningitis patient, 26 (52\%) cases were undernutritional condition. In case of pyogenic meningitis $13(48.14 \%)$ cases are undernourished, in tuberculous $7(78 \%)$ patient and in viral meningitis $6(42.85 \%)$ patients are undernourished. Colour of CSF was turbid in maximum pyogenic meningitis patients and clear nearly in all viral cases. In this series CSF glucose concentration was very low $32.8 \mathrm{mg} / \mathrm{dl}$ and protein concentration was high $197.6 \mathrm{mg} / \mathrm{dl}$ in case of pyogenic cases. In viral cases glucose and protein were normal. This findings correlates with hospital based study of Bangladesh. ${ }^{13}$ In a study of CSF analysis- acute bacterial versus viral meningitis done in Dubi, United Arab Amirat from 2005-07, CSF Glucose was found to be very low in bacterial than viral meningitis where mean CSF glucose concentration was $26.50 \mathrm{mg} / \mathrm{dl}( \pm 21.56)$ Vs $67.00( \pm 18.96) \mathrm{mg} / \mathrm{dl} .{ }^{19}$

CSF cell counts were very high (average $>4200 / \mathrm{mm} 3$ ) in case of pyogenic meningitis of which most were neutrophils (87\%) but in cases of tuberculour meningitis cell counts were very low (average 223/mm3) of which most were lymphocyts (76\%). This finding correlates with the findings of maximum authorities. ${ }^{20}$

Study shows that in $55.55 \%$ of the tuberculous and $51.85 \%$ of pyogenic meningitis cases duration of hospital stay was between $1-2$ weeks duration. Hospital stay for more than two weeks was proportionately much higher in tuberculous type $(44.44 \%)$ compared to pyogenic $(25.92 \%)$ and viral/aseptic type (7.14\%). Total 5 (10\%) patients expired out of 50. Total 16 patients were comatose and 4 (25\%) of them died. On the other hand normal and confused patients were 9 with out any death. Out of 25 lethargic patients 1 (4\%) expired. So death was high in unconscious patients. Mortality was high in viral cases $21.43 \%$ compared to pyogenic $(7.40 \%)$ cases. These findings correlate with other studies. In another study mortality rate in untreated HSE is around $70 \%, 40 \%$ to $70 \%$ in Nipah virus encephalitis. ${ }^{27}$

Gradual communication of the truth within the context of continued support and encouragement almost always leads to enhanced hope. Breaking bad news generally causes distress to both the patient and his/her family. In this issue we obtain the response from the attendance by consultation, counseling, open question and elicit the feelings, emotion and concern. We found that only $14 \%$ of attendance carries clear idea regarding the disease, but majority of patient attendance lack of concept about the disease, its management and prognosis on future time.

\section{CONCLUSION}

With this shortcoming this study is designed that may help us to understand the Clinoco-pathological issue of meningitis with management and influence us to better concern to control the disease thereby rescuing some people from long term disability and or death and preventing the burden of the family and the country.

\section{REFERENCES}

1. Sáez-Llorens X, McCracken GH. "Bacterial meningitis in children".Lancet 2003; 361:2139-48

2. Ginsberg L. "Difficult and recurrent meningitis". Journal of Neurology, Neurosurgery, and Psychiatry 2004; 75 (1): $16-21$

3. Logan SA, MacMahon E ."Viral meningitis". BMJ 2008; $336: 36-40$

4. Attia J, Hatala R, Cook DJ, Wong JG . "The rational clinical examination. Does this adult patient have acute meningitis?". Journal of the American Med Asso.1999; 282 (2): 175-81.

5. World health organization . "Detecting meningococcal meningitis epidemics in highly-endemic African countries". Weekly Epidemiological Record 2000;75 (38): 306-9

6. Gurley ES1, Hossain MJ, Montgomery SP, Petersen LR, Sejvar JJ, Mayer LW, Whitney A, Dull P, Nahar N, Uddin AK, Rahman ME,Ekram AR, Luby SP, Breiman RF. "Etiologies of bacterial meningitis in Bangladesh: results from a hospital-based study". Bangladesh (ICDDR,B), Dhaka, Bangladesh

7. World Health Organization . Control of epidemic meningococcal disease, practical guidelines, 2nd edition, WHO/EMC/BA/98 (PDF). 1999; 3:1-83

8. World health organization . "Detecting meningococcal meningitis epidemics in highly-endemic African countries" (PDF).Weekly Epidemiological Record 2003;78 (33): 294-6

9. Van de Beek D, de Gans J, Tunkel AR, Wijdicks EF . "Community-acquired bacterial meningitis in adults". The New Engl J of Med 2006; 354 (1): 44-53 
10. Kanegaye J.T., Soliemanzadeh P., Bradley J.S. Lumbar puncture in pediatric bacterial meningitis: defining the time interval for recovery of cerebrospinal fluid pathogens after parenteral antibiotic pretreatment. Pediatrics 2001;108: 1169-74

11. Tunkel A.R., Hartman B.J., Kaplan S.L., et al. Infectious Disease Society of America practice guidelines for management of bacterial meningitis. Clin. Infect. Dis. 2004;39:1267-84

12. P. Madhumita, N Gputa, Depantent of Medicine, Maulana Azed Medical college Hospital, India. Clinical \& Bacteriological spectrum of community-acquired acute Bacterial Meningitis in Adults at a tertiary care hospital in northern India. Int. J Nutr Pharmacol Neurol Dis 2001; (2): 194-0

13. Emily S. Gurley, M, Jahangir Hossain, Susan P. Montgomery, Lyle R. Petersen, James J. Sejvar, Leonard W. Mayer, Anne Whitney et.al. Etiologies of Bacterial Meningitis in Bangladesh: Results from a Hospital-Based Study Am. J. Trop. Med. Hyg. 2009;81(3):475-83

14. ICDDRB. Surveillance for encephalitis in Bangladesh 2009

15. Pfister HW, Feider W, Ein hauple KM. Spectrum of Complications during bacterial meningitis in adults: Arch Neurol 1993; 50: 575-81

16. Van be Beek D. de Cans J, S panjarrd L, Weisfelt M, Reitsma J, B Vermeulen M; Clinical Features and bacterial meningitis. N Engl. J Med 2004:351:1849-59

17. Sarkari NB, Thacker AK. Barthwal SP, Mishra VK, prapann S, Srivastava D. et.al. Japanese Encephalitis part-1 clinical profile of 1,282 adult acute cases of four epidemics. J Neurol. 2011 June 17

18. Aronin SI, peduzzip, Quagliarello VJ. Community acquired bacterial Meningitis: risk Stratification for adverse clinical outcome and effect of antibiotic timing. Ann Intern Med. 1998; 129:862-9

19. Abro AH, Addou AS, Ali H, Ustadiam, Hasab AAH, CSF Analysis-Acute bacterial versus viral Meningitis Pak J, Med Sci 2008;24(5):645-50

20. D.A warrel and J.J Farrar, Viral infections of the central nervous system, in Oxford Text Book of Medicine 3rd edition, 1999 University press, Oxford (UK). PP 1129- 40

21. L.-M. Tang, S.-T. Chen, W.-C, Hsu and R.-K Lyu Acute bacterial Meningitis in adults : a Hospital based epidemiological study. QJM September 1999
;92(12).719-25

22. G.E Thwaites, TTH chau, K stepniewska, N H Phu, LV Chuong, DX Sinh, et. al. Diagnosis of adult tuberculous meningitis by use of clinical and laboratory feature. The lancet 2002;360:1287- 92

23. H El Bashir, M Laundy, R Booy, Diagnosis and Treatment of bacterial meningitis, Arch Dis Child 2003; 88: 615-0

24. Ragu naihan L, Ramsay M, Bornow R, Guiver M, Grays, et.al. Meningococeal Meningitis: survey report Withington Hospital,Manchester UK. J. Infect. $2000 ; 40(1): 74-9$.

25. Kanegaye JT, Soliemanzadeh, Bradley J, Perdiatries, 2001;108 (5): 1169

26. Geiseler PJ, Nelsom KE, Levin S, Reddi KT, Moses VK, community acquired pruient meningitis: a review of 1,316 cases during antibiotic era. Rev Infect Dis. 1980; 2 (5): 725 27. Joel M Montogomery, Mohamed J. Hossain et al, Risk Factors for Nipah Virus Encephalitis in Bangladesh, Emergency Infectious Diseases 2008;14:10 